\title{
Stage-selective differential expression of kinesin (Kif) genes in cortical and medullary epithelial cells of the murine thymus.
}

\author{
Shahan Mamoor ${ }^{1}$ \\ 1Thomas Jefferson School of Law \\ shahanmamoor@gmail.com \\ San Diego, CA 92101
}

The thymus is an organ with immunologic functions conserved from mouse to man (1). Both fetal and mammalian thymuses exist (1). The processes of negative and positive selection (2) both occur in the thymus and are thought to be conducted in an anatomically distinct fashion, across development. We performed global differential gene expression profiling using a microarray dataset (6) of the murine cortical epithelial cell (cTEC) and medullary epithelial cell (mTEC) transcriptome at months 1, 3, and 6. This analysis revealed that multiple members of the kinesin (Kif) gene family were differentially expressed by cTECs and by MTEC that express high levels of the class II major histocompatibility complex, in a stage-selective manner, but not by mTEC that express low levels of the class II major histocompatibility complex. The products of Kif genes may serve some important motor or transport function in the cortical or medullary epithelium of the mammalian thymus.

Keywords: thymus, cTEC, cortical thymic epithelial cells, mTEC, medullary thymic epithelial cells, kinesin, Kif, differential gene expression, systems-level analysis of the immunologically active cells of the thymus. 


\section{Introduction}

The immune system is a military of cells that must be able to discern what is self in order to prevent autoimmune damage to the human body. The thymus, unique in the mammalian body, possesses the ability to educate developing lymphocytes what it means to be self by presenting them with antigen that is generally otherwise restricted to peripheral organs (tissue restricted antigens) (1-4). Antigen presentation by the thymus for instruction of self to developing lymphocytes occurs though the epithelial cells in the cortex and medulla of the thymus, termed cTEC and mTEC (5). Antigen presentation on these cells by expression of the Class II major histocompatibility complex peptide antigen receptor MHC-II is central to this process $(7,8)$. mTEC expressing high levels of MHC-II (mTEChi) also express relatively higher levels of the autoimmune regulator Aire, which induces promiscuous expression of tissuerestricted antigen for self-antigen presentation, for negative selection (8). cTEC, different from mTEC, are involved in positive, rather than negative selection of T-lymphocytes $(9,10)$.

To determine the most significant changes in the transcriptional behavior of cTEC and mTEC during mammalian development, here we used a public microarray dataset (6) containing transcriptome data from cTEC, as well as mTEC expressing high or low levels of the MHC-II complex (mTEChi or mTEClo) isolated from the thymus of mammalian mice at 1 month, 3 months, and 6 months of age, by global differential gene expression profiling. This transcriptome study revealed that transcripts from the kinesin gene family are among the most differentially expressed genes when comparing the cTEC and mTEChi transcriptomes across mammalian development suggests a potential cell-type specific role for the products of these kinesin genes in the cortical and medullary epithelial cells of the mammalian thymus.

\section{Methods}

GSE56928 was utilized for this differential gene expression analysis, performed using GEO2R. This public dataset (6) was generated by the authors using microarray (Affymetrix Mouse 4302.0 array) of cTEC, mTEChi and mTEClo , $n=2$ for each developmental time point (1 
month, 3 month, and 6 months). The "Benjamini and Hochberg" method of $p$-value adjustment was utilized for $p$-value calculation by GEO2R, and the "Auto-detect" option was used for logtransformation applied to global expression data. The NCBI generated category of platform annotation was used. For statistical testing to determine the significance of difference between average RNA expression values of each kinesin gene between cTEC and between mTEChi at each time point, an ordinary one-way ANOVA with multiple comparisons (Tukey's multiple comparisons test) was utilized (PRISM 8.1.2) (227).

\section{Results}

Kinesin superfamily genes are among the most differentially expressed in the cTEC transcriptome when comparing the global gene expression profiles of cTECs across mammalian development

We performed global differential gene expression profiling using a public microarray dataset containing transcriptome data from cTEC at three developmental time points in murine mammalian life: 1 month, 3 months, and 6 months (6). This analysis revealed that transcripts from 5 kinesin genes were among the most differentially expressed in the cTEC transcriptome when comparing cTECs at these developmental stages. These included Kif2c, Kif11, Kif5c, Kif13a and Kif13b (Table 1).

Out of 45101 total transcripts detected, Kif2c was the 30th most differentially expressed gene when comparing the global gene expression profiles of cTEC at 1 month, 3 months, and 6 months (Table 1; $p=8.2 \mathrm{E}-06$ ). Kif11 was 60th most differentially expressed (Table 1; $p=1.49 \mathrm{E}-05)$; Kif5c was the $122 \mathrm{nd}$ most differentially expressed (Table $1 ; p=5.83 \mathrm{E}+01)$, Kif13a was the 171st most differentially expressed gene (Table 1; $p=5.05 \mathrm{E}-05)$, and Kif13b was the 186th most differentially expressed gene in the cTEC transcriptome across the 1 month, 3 month, and 6 months in mammalian mammalian life (Table $1 ; p=5.83 \mathrm{E}-05$ ).

Over mammalian life in the cortical epithelial cells of the thymus, the expression of two of these differentially expressed Kif genes, Kif2c and Kif11 decreased, while the expression of the three other differentially expressed Kif genes, Kif13a, Kif13b and Kif5c increased. 
Kif2c expression decreased from 1 month to 6 months, and this change was statistically significant (Figure 1; $p=0.0031$ ). Kif2c expression also decreased from 1 month to 3 months (Figure $1 ; p=0.0031$ ) but did not significantly change from 3 months to 6 months (Figure 1; $\mathrm{p}=0.8609$ ).

Kif11 expression also decreased from 1 month to 6 months (Figure $1 ; p=0.0206$ ). The expression of Kif11 decreased even more significantly from 1 month to 3 months (Figure 1; $p=0.0042$ ). From 3 months to 6 months, however, the expression of Kif11 in cTEC increased to a level below that of CTEC at 1 month but above that of CTEC at 3 months (Figure $1 ; p=0.0206$ ).

Kif13a expression in the cortical epithelial cells of the thymus increased from 1 month to 6 months (Figure 1; $p=0.0093$ ) while it decreased from 1 month to 3 months (Figure 1; $\mathrm{p}=0.0093$ ). From 3 months to 6 months, however, Kif5c expression in cTEC increased to a level even higher than that at 1 month (Figure $1 ; p=0.0025$ ).

Kif13b expression in cTEC increased from 1 month to 6 months (Figure $1 ; p=0.0139$ ) but did not significantly change from 1 month to 3 months (Figure $1 ; p=0.8825$ ). This increase over mammalian life of Kif13b expression in cTEC occurred primarily between 3 to 6 months (Figure $1 ; p=0.0139$ ).

Kif5c expression in the epithelial cells of the cortex of the thymus increased from 1 month to 6 months $(p=0.0017)$, and this change primarily occurred between 3 months to 6 months (Figure $1 ; p=0.0017$ ). The expression of Kif5c did not significantly change between 1 month to 3 months (Figure $1 ; p=0.3676$ ).

Kinesin superfamily genes are among the most differentially expressed in mTEChi, but not mTEClo transcriptome when comparing the global gene expression profiles of mTECs across mammalian development

We performed a similar global differential gene expression profiling using the same microarray dataset, now with transcriptome data from mTEChi at the same three developmental time points in murine mammalian life: 1 month, 3 months, and 6 months (6). Here, 3 transcripts 
from the Kif gene family were among the most differentially expressed in the mTEChi transcriptome when comparing mTEChi at these developmental stages. These included Kif5c, Kif13b, and Kif20b (Table 1).

Kif13b was the 56th most differentially expressed gene out of 45101 total transcripts in the mTEC $^{\text {hi }}$ transcriptome detected and measured in the microarray dataset used for this study (Table $1 ; p=1.77 \mathrm{E}-05)$. Kif5c was the 115th most differentially expressed gene in the mTEChi transcriptome (Table 1; $p=4.23 \mathrm{E}-05$ ), and Kif20b was the 146th most differentially expressed gene when comparing mTEChi transcriptomes between 1 month, 3 months and 6 months (Table 1; $p=6.19 \mathrm{E}-05)$

All three differentially expressed kinesin genes, Kif5c, Kif13b and Kif20b increased in expression level in mTEChi over mammalian development. Kif5c increased significantly from 1 to 6 months (Figure $2 ; p=0.0017$ ) and from 3 to 6 months (Figure $2 ; p=0.0016$ ) but not from 1

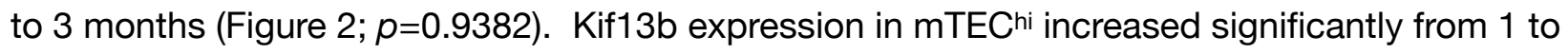
6 months (Figure $2 ; p=0.0008$ ), as well as from 1 to 3 months (Figure $2 ; p=0.0488$ ) and 3 to 6 months (Figure $2 ; p=0.0018$ ). Kif20b expression increased from 1 to 6 months (Figure $2 ; p=$ 0.0129) and from 3 to 6 months (Figure 2; $p=0.0148$ ) but not from 1 to 3 months (Figure 2; $p=0.9399)$.

There were no kinesin genes represented in the two hundred and fifty most differentially expressed genes in the mTEClo transcriptome. The highest ranked differentially expressed Kif gene in the mTEClo transcriptome when comparing gene expression profiles at 1 month, 3 months and 6 months was Kif1b, ranked 251 out of 45101 total transcripts. In this study, we collected RNA expression values only for genes whose expression was ranked 250/45101 or higher.

Kif5c and Kif13b were the only genes whose expression significantly changed at the level of the transcriptome in both cTEC and mTEChi across mammalian development, and their expression levels increased significantly from 1 to 6 months of mammalian life in mice. 


\section{Discussion}

In this study, we performed global differential gene expression profiling of both cortical and medullary epithelial cells of mice at three time points in mammalian development: 1 month, 3 months, and 6 months of age. When comparing the global gene expression profiles of cTEC at these three stages of development, we found that 5 kinesin genes were among the most differentially expressed genes in the cTEC transcriptome. These genes included Kif2c, Kif11, Kif5c, Kif13a and Kif13b.

The products of Kif genes are motor proteins involved in the mechanical processes by which intracellular cargoes including membranous organelles, mRNA, macromolecular protein complexes are transported using the microtubule cytoskeleton as a track, and energy (ATP) towards the plus-end of microtubules $(11,12)$. Kinesins are important for processes such as mitosis, where they form contacts with the kinetochore and orchestrate spindle assembly (11). There exist kinesin families 1, 2, and 3, which differ in their domain structure (11). Transporting large cellular cargoes requires the use of energy and force, and kinesins can accomplish this using some combination, depending on the Kinesin gene type, of a motor domain that interacts with microtubules and a domain that interacts with the cargo to be transported (11).

A previous study found that Kif7 was required for expression of MHC-Class II on the mTEC surface, and that Kif7 deficient thymuses displayed increases in the CD44+CD25+ CD4CD8- thymocyte population and a concurrent decrease in double positive CD4+CD8+ cells, as well as a decrease in mature CD8+ lymphocytes (13). Kif7 deficient thymocytes also displayed defects in Hedgehog signaling as they expressed higher levels of Hedgehog targeted gene patched1 than wild-type thymocytes, yet were less responsive to treatment with recombinant Sonic Hedgehog (Shh) ligand (13). The Kif genes identified in this analysis as developmentally differentially expressed in the cortical epithelial cells of the thymus have not yet been studied in this manner.

In summary, we found here that multiple genes from the Kinesin family, including Kif2c, Kif11, Kif5c, Kif13a and Kif13b in cTEC, and Kif5b, Kif13b and Kif20b in mTEChi, were 
differentially expressed in a stage-specific manner from 1 to 6 months of murine mammalian life. It is not clear what function stage-specific increases in Kif5c, Kif13a or Kif13b gene expression, or decreased in Kif2c and Kif11 serve in the epithelial cortex of the mammalian thymus, nor is it understood what role stage-specific increases in Kif5c, Kif13b and Kif20 gene expression serve in the medulla of the mammalian thymus. Specific deletion of each of these kinesin genes, individually, in the thymus using $\beta 5 t-C r e$ and subsequent in vivo analysis of thymocyte development, in conjunction with ex vivo analysis of lymphocyte function (cytokine secretion, antigen recall) can contribute to the characterization of a potential role for these motor proteins in the immunologically active cTEC and mTEChi. 


\section{References}

1. Anderson, M.S., Venanzi, E.S., Klein, L., Chen, Z., Berzins, S.P., Turley, S.J., Von Boehmer, Miller, J.F.A.P. and Mitchell, G.F., 1969. Thymus and antigen-reactive cells. Immunological Reviews, 1(1), pp.3-42.

2. Surh, C.D. and Sprent, J., 1994. T-cell apoptosis detected in situ during positive and negative selection in the thymus. Nature, 372(6501), p.100.

3. H., Bronson, R., Dierich, A., Benoist, C. and Mathis, D., 2002. Projection of an immunological self shadow within the thymus by the aire protein. Science, 298(5597), pp. 1395-1401.

4. Hogquist, K.A., Baldwin, T.A. and Jameson, S.C., 2005. Central tolerance: learning selfcontrol in the thymus. Nature Reviews Immunology, 5(10), p.772.

5. Rossi, S.W., Jenkinson, W.E., Anderson, G. and Jenkinson, E.J., 2006. Clonal analysis reveals a common progenitor for thymic cortical and medullary epithelium. Nature, 441 (7096), p.988.

6. Ki, S., Park, D., Selden, H.J., Seita, J., Chung, H., Kim, J., lyer, V.R. and Ehrlich, L.I., 2014. Global transcriptional profiling reveals distinct functions of thymic stromal subsets and agerelated changes during thymic involution. Cell reports, 9(1), pp.402-415.

7. Gray, D., Abramson, J., Benoist, C. and Mathis, D., 2007. Proliferative arrest and rapid turnover of thymic epithelial cells expressing Aire. Journal of Experimental Medicine, 204(11), pp.2521-2528.

8. Hinterberger, M., Aichinger, M., Da Costa, O.P., Voehringer, D., Hoffmann, R. and Klein, L., 2010. Autonomous role of medullary thymic epithelial cells in central CD4+ T cell tolerance. Nature immunology, 11(6), p.512.

9. Murata, S., Takahama, Y. and Tanaka, K., 2008. Thymoproteasome: probable role in generating positively selecting peptides. Current opinion in immunology, 20(2), pp.192-196.

10. Gommeaux, J., Grégoire, C., Nguessan, P., Richelme, M., Malissen, M., Guerder, S., Malissen, B. and Carrier, A., 2009. Thymus-specific serine protease regulates positive selection of a subset of CD4+ thymocytes. European journal of immunology, 39(4), pp. 956-964.

11. Verhey, K.J., Kaul, N. and Soppina, V., 2011. Kinesin assembly and movement in cells. Annual review of biophysics, 40, pp.267-288.

12. Miki, H., Setou, M., Kaneshiro, K. and Hirokawa, N., 2001. All kinesin superfamily protein, KIF, genes in mouse and human. Proceedings of the National Academy of Sciences, 98(13), pp.7004-7011.

13. Lau, C.I., Barbarulo, A., Solanki, A., Saldaña, J.I. and Crompton, T., 2017. The kinesin motor protein Kif7 is required for T-cell development and normal MHC expression on thymic epithelial cells (TEC) in the thymus. Oncotarget, 8(15), p.24163. 

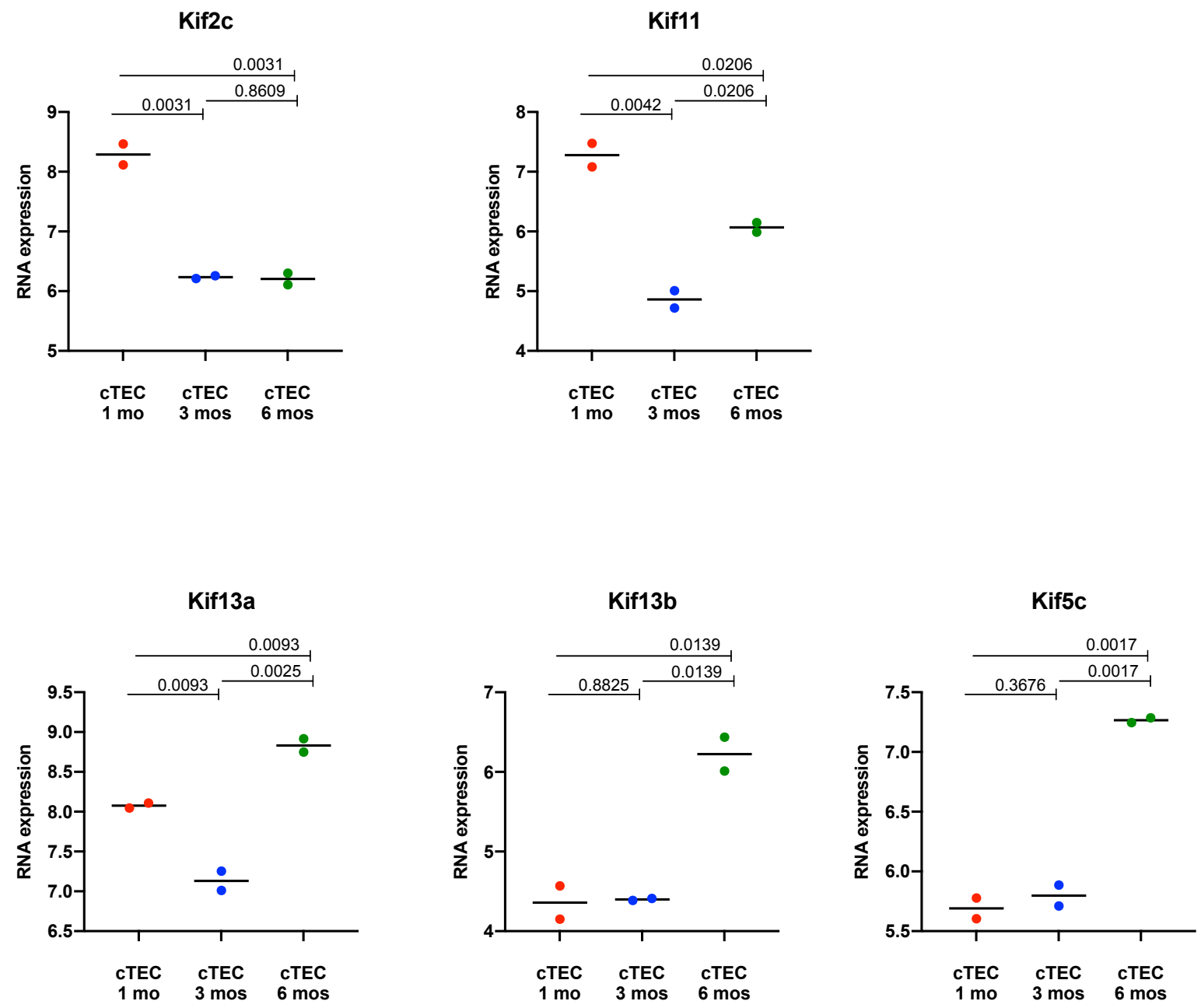

Figure 1: Kinesin family genes are among the most differentially expressed in the cTEC transcriptome when comparing the global gene expression profiles of cTECs across mammalian development

The expression of kinesin genes Kif2c, Kif11, Kif13a, Kif13b and Kif5c is graphically represented, with the mean value of RNA expression of each gene at each time point shown as a black horizontal bar. A statistical test to evaluate the significance of the difference of RNA expression level between each developmental time point was performed using an ordinary one-way ANOVA with multiple comparisons (Tukey's multiple comparisons test) and the $p$ value for each comparison is denoted on each graph. 

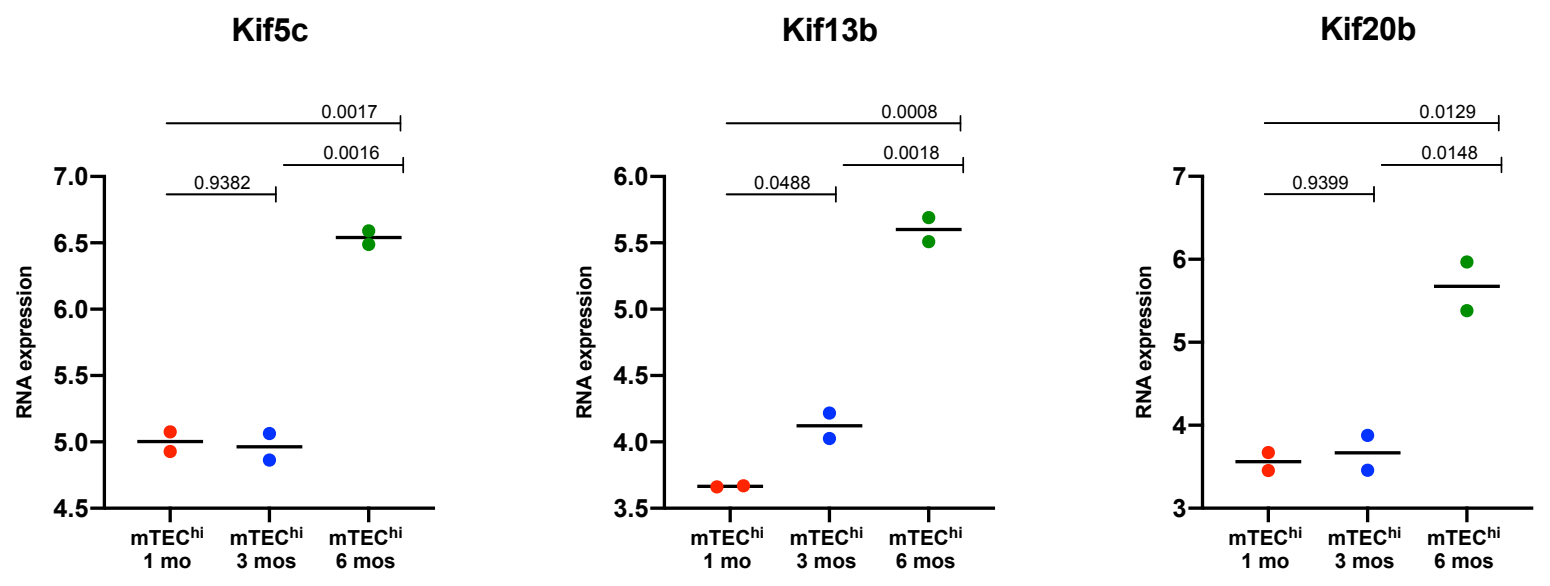

Figure 2: Kinesin superfamily genes are among the most differentially expressed in the mTEChi transcriptome when comparing the global gene expression profiles of mTEChi $^{\mathrm{h}}$ across mammalian development.

The expression of kinesin genes Kif5b, Kif13b and Kif20b in the medullary epithelial cells of the thymus expressing high levels of MHC Class II is graphically represented, with the mean value of RNA expression of each gene at each time point shown as a black horizontal bar. A statistical test to evaluate the significance of the difference of RNA expression level between each developmental time point was performed using an ordinary one-way ANOVA with multiple comparisons (Tukey's multiple comparisons test) and the $p$-value for each comparison is denoted on each graph. 
Table 1

\begin{tabular}{|r|l|l|r|r|l|}
\hline Rank & Gene Symbol & Gene Name & p-value & \multicolumn{1}{l|}{ ID } \\
\hline $\mathbf{3 0}$ & Kif2c & kinesin family member 2C & $8.2 \mathrm{E}-06$ & $8.57 \mathrm{E}+01$ & 1437611_x_at \\
\hline $\mathbf{6 0}$ & Kif11 & kinesin family member 11 & $1.49 \mathrm{E}-05$ & $7.23 \mathrm{E}+01$ & 1435306 _a_at \\
\hline $\mathbf{1 2 2}$ & Kif5c & kinesin family member 5C & $5.83 \mathrm{E}+01$ & $5.83 \mathrm{E}+01$ & 1422945 a_at \\
\hline $\mathbf{1 7 1}$ & Kif13a & kinesin family member 13A & $5.05 \mathrm{E}-05$ & $5.08 \mathrm{E}+01$ & 1447853_x_at \\
\hline $\mathbf{1 8 6}$ & Kif13b & kinesin family member 13B & $5.83 \mathrm{E}-05$ & $4.87 \mathrm{E}+01$ & 1453276_at \\
\hline & & & & & \\
\hline $\mathbf{5 6}$ & Kif13b & kinesin family member 13B & $1.77 \mathrm{E}-05$ & $6.14 \mathrm{E}+01$ & 1453276_at \\
\hline $\mathbf{1 1 5}$ & Kif5c & kinesin family member 5C & $4.23 \mathrm{E}-05$ & $4.82 \mathrm{E}+01$ & 1422945_a_at \\
\hline $\mathbf{1 4 6}$ & Kif20b & kinesin family member 20B & $6.19 \mathrm{E}-05$ & $4.34 \mathrm{E}+01$ & 1440924_at \\
\hline & & & & & \\
\hline
\end{tabular}

Table 1: Multiple kinesin family genes are differentially expressed in the cortical and medullary epithelial cells of the thymus.

The rank of differential expression of each kinesin gene relative to 45101 total transcripts detected and measured, $p$-value of differential expression relative to the cTEC transcriptomes, F statistic, (all determined by NCBI GEO2R) and Affymetrix probe ID for each transcript are displayed. Above (ranked 30-186): cTEC. Below (ranked 56-146): mTEChi. 